\title{
Une organisation des flux au sein des urgences ${ }^{1}$
}

\section{An organization of flows within the emergency}

\author{
D. Pateron \\ (C) SFMU et Springer-Verlag France 2013
}

Plusieurs études ont montré que plus d'un service d'urgence sur deux fonctionnent en suractivité, c'est-à-dire qu'ils ne pouvaient pas, de façon répétée, prendre en charge les malades dans les conditions optimales et sans délai d'attente prolongé. L'allongement du temps de prise en charge médicale retentit sur la qualité des soins, la satisfaction des patients et la « productivité » des professionnels de l'urgence. L'incapacité des services de prendre en charge rapidement les malades n'est pas constante et les périodes de suractivités dans la journée et dans la semaine sont assez reproductibles. Les problèmes à résoudre sont multiples pour améliorer cette situation. Ils concernent l'amont et surtout l'aval des services d'urgences. Cependant, l'organisation interne des services influence également la fluidité de passage des patients. Cette organisation doit répondre à des critères réglementaires et des bonnes pratiques recommandées par les sociétés savantes. Des pistes d'amélioration de l'organisation ont été proposées et certaines ont été étudiées par une démarche scientifique d'évaluation [1]. Elles nécessitent le plus souvent la concentration de moyens sur certaines filières de prise en charge dont les effets retentissent sur la fluidité de l'ensemble du service. Elles s'inscrivent dans le parcours du patient au sein de la structure des urgences qui comprend sa présentation initiale, son enregistrement, le tri du patient, l'évaluation médicale et l'orientation du malade en particulier en cas d'hospitalisation.

La présence d'une infirmière organisatrice de l'accueil (IOA) est une recommandation professionnelle bien qu'elle ne fasse pas partie des obligations des derniers décrets [2].

\section{Pateron $(\bowtie)$}

Service des urgences, CHU Saint-Antoine,

Assistance publique-hôpitaux de Paris, université Pierre et Marie Curie-Paris 6,

184, rue du Faubourg Saint-Antoine, F-75012 Paris, France

e-mail : dominique.pateron@sat.aphp.fr

\footnotetext{
${ }^{1}$ Texte présenté dans le cadre des $1^{\text {res }}$ Assises de l'urgence, 13 septembre 2012, Paris. L'ensemble des textes est disponible sur http:// www.samu-de-france.fr/documents/actus/129/732/sudf_1-assisesde-1-urgence_txt-et-reco_120913_vf.pdf(dernier accès le 12 février 2013)
}

Elle joue un rôle essentiel dans le tri de gravité et donc dans l'attente des patients avant d'accéder à l'évaluation médicale.

Le rôle de l'architecture des services est important dans la mesure où il doit conjuguer la surveillance des patients présents sur le site et les différentes étapes de la prise en charge. La SFMU a émis des recommandations précises [3]. La notion de marche en avant évitant le retour du patient vers une zone déjà empruntée est intéressante. Malheureusement les délais de construction ou de rénovation des services après leur conception sont de plusieurs années et les recommandations ou concepts sur lesquelles elles se sont appuyées sont parfois caduques.

Filière rapide de prise en charge

Elle concerne les malades ne présentant pas un risque vital immédiat et n'ayant pas besoin d'examen complémentaires nécessitant un délai important ( $>1$ heure). Cette prise en charge rapide des malades les moins lourds peut paraître paradoxale dans la mesure où elle accélère la prise en charge des malades qui en aurait le moins besoin. Les études ont montré que la fluidité globale du service s'améliorait profitant ainsi à l'ensemble des patients. Cette filière s'applique particulièrement à la traumatologie courante.

\section{Assoir les patients qui peuvent l'être}

L'arrivée des patients en ambulance ou amenés par les pompiers se fait presqu'exclusivement en position couchée. Le maintien du malade sur un brancard alors que son état ne le justifie pas est source d'une mobilisation plus contraignante, consommant du temps paramédical inutile.

Prise en charge protocolisée de certaines pathologies

Les pathologies concernées doivent être repérées facilement. L'IOA n'est pas sensé faire de diagnostic. Cette prise en charge concerne la prise en charge de la douleur, la prescription anticipée de radiographie, fondée sur des protocoles de service et sous couvert d'un recours possible permanent au médecin.

\section{Mise en place d'un médecin référent}

Cette solution nécessite la mobilisation d'un médecin et ne peut être appliquée que lorsque le flux est tel qu'il justifie la présence de plusieurs médecins au SAU. La fiche de mission de ce poste doit être précise et l'implication et 
l'adaptation du médecin à ce poste sont nécessaires. Il doit participer au tri, à l'orientation rapide du malade vers la radiographie et la prise en charge de certains malades tout en assurant la coordination du service et la relation médicale avec l'environnement du service.

Amélioration des délais de réalisation des examens complémentaires

Le délai d'obtention des examens complémentaires est un élément important du temps de passage. Une simplification du circuit d'obtention de l'examen ou des appareils dédiés à la prise en charge des malades urgents concourent à sa réduction. Il s'agit des examens radiologiques en particulier le scanner et l'IRM et les examens de biologie délocalisée.

\section{Informatisation du parcours patient}

Elle concerne l'enregistrement, l'horodatage de différentes phases du parcours et la mise en place de sentinelles informatiques.

\section{Adaptation des effectifs au flux}

Cette adaptation passe par une connaissance précise des flux dans la journée et sur la semaine et selon les périodes de l'année. Elle concerne également l'arrivée d'un afflux important et inopiné de patients. Cette adaptation nécessite une « flexibilité » du travail parfois difficile à conjuguer avec les conditions de travail déjà pénibles des personnels des urgences. Cette adaptation des personnels peut se conjuguer avec une modulation des zones de soins.

\section{Hospitalisations anticipées}

Certaines hospitalisations nécessaires mais non urgentes pourraient être anticipées, mais en l'absence d'une procé- dure efficace, les patients passent le plus souvent par les urgences. L'organisation d'un réseau impliquant les médecins généralistes, les services hospitaliers et l'évaluation des lits disponibles dans l'hôpital pourrait permettre d'attribuer le plus rapidement possible le lit adéquat. Ceci permettrait une meilleure occupation des lits disponibles en évitant un passage inutile aux urgences. Un PHRC est actuellement en cours pour tester l'hypothèse du bénéfice d'une telle procédure. Un raisonnement similaire peut concerner les consultations spécialisées.

Ce dernier exemple montre que le service des urgences n'est pas l'hôpital dans l'hôpital mais qu'une bonne fluidité de nos structures dépend avant tout de l'implication de l'hôpital dans son ensemble.

\section{Références}

1. Wiler JL, Gentle C, Halfpenny JM, et al (2010) Optimizing emergency department front-end operations. Ann Emerg Med 55: 142-60

2. Société française de médecine d'urgence (2004) Infirmière organisateur de 1'accueil, référentiel SFMU : http://www.sfmu.org/ documents/ressources/referentiels/ioa2004.pdf (dernier accès le 13 février 2013)

3. Société française de médecine d'urgence (2004) Architecture des services d'urgences, Recommandations de la SFMU http:// www.sfmu.org/documents/ressources/referentiels/recom_sfmu_archi_ service_urg.pdf (dernier accès le 13 février 2013) 